\title{
HAUSDORFF MEASURE AND LEVEL SETS OF TYPICAL CONTINUOUS MAPPINGS IN EUCLIDEAN SPACES
}

\author{
BERND KIRCHHEIM \\ In memory of Hana Kirchheimová
}

\begin{abstract}
We determine the Hausdorff dimension of level sets and of sets of points of multiplicity for mappings in a residual subset of the space of all continuous mappings from $\mathbb{R}^{n}$ to $\mathbb{R}^{m}$.
\end{abstract}

Questions about the structure of level sets of typical (i.e. of all except a set negligible from the point of view of Baire category) real functions on the unit interval were already studied in [2], [1], and also [3]. In the last one the question about the Hausdorff dimension of level sets of such functions appears. The fact that a typical continuous function has all level sets zero-dimensional was commonly known, although it seems difficult to find the first proof of this. The author showed in [9] that in certain spaces of functions the level sets are of dimension one typically and developed in [8] a method to show that in other spaces functions are typically injective on the complement of a zero dimensional set-this yields smallness of all level sets. Here we are going to extend this method to continuous mappings between Euclidean spaces and to determine the size of level sets of typical mappings and the typical multiplicity in case the level sets are finite; see Theorems 1 and 2.

We will use the following notation. For $M$ a set and $\varepsilon>0, U(M, \varepsilon)$, resp. $B(M, \varepsilon)$, is the open, resp. closed, $\varepsilon$-neighborhood of $M$ and we write $U(x, \varepsilon)$ instead of $U(\{x\}, \varepsilon)$. Given a metric space $X$ of functions, we say that typical $f \in X$ has property $P$ if $\{f \in X$; non $P(f)\}$ is first category in $X$. We will always deal with spaces of continuous mappings equipped with the supremum metric. Finally, to prove that certain properties are typical, we will use the Banach-Mazur game. Let $(X, \rho)$ be a metric space and $M \subset X$. In the first step Player A selects an open ball $U\left(x_{1}, \varepsilon_{1}\right)$. In the second step Player B selects an open ball $U\left(x_{2}, \varepsilon_{2}\right) \subset U\left(x_{1}, \varepsilon_{1}\right)$ and then $A$ continues with $U\left(x_{3}, \varepsilon_{3}\right) \subset$ $U\left(x_{2}, \varepsilon_{2}\right)$, and so on. By definition Player B wins if $\bigcap_{j=1}^{\infty} B\left(x_{j}, \varepsilon_{j}\right) \subset M$, else Player A wins. We have the following

There is a winning strategy for Player B if and only if $X \backslash M$ is a first category set in $X$.

Received by the editors January 5, 1994; originally communicated to the Proceedings of the AMS by Andrew Bruckner.

1991 Mathematics Subject Classification. Primary 46E15, 26B99, 28 A78.

Key words and phrases. Level set, space of continuous mappings, Hausdorff dimension. 
For the proof see [10]. We will also assume the definition of Hausdorff measure and dimension, also for more general Hausdorff functions $\phi$; see e.g. [11].

Theorem 1. Let $1 \leq n<m$ be given. For any $k \geq 1$ we denote $d_{k}=m-$ $k(m-n)$. Then for typical continuous $f: B\left(0,1 ; \mathbb{R}^{n}\right) \rightarrow \mathbb{R}^{m}$ and any $k \geq 2$ the set

$$
M_{k}(f)=\left\{x \in B(0,1) ; \operatorname{card} f^{-1}(f(x)) \geq k\right\}
$$

as well as the set $f\left(M_{k}(f)\right)$ are $F_{\sigma}$-sets of Hausdorff dimension $d_{k}$; moreover for any nonvoid open $U \subset B(0,1)$ both sets $U \cap M_{k}(f)$ and $f\left(M_{k}(f) \cap U\right)$ are of non- $\sigma$-finite $d_{k}$-dimensional measure. For $k=1$ the statements concerning $f\left(M_{1}(f) \cap U\right)$ remain true.

For the proof of this theorem we need some preparation. Until the end of its proof we will therefore always (unless explicitly stated in another way) assume that $n, k \geq 1, m>n$, that $d_{k}$ and $M_{k}(f)$ are defined as above, and that the following four orthogonal projections are given.

- $P: \mathbb{R}^{n} \rightarrow \mathbb{R}^{d_{k}}, P\left(x_{1}, \ldots, x_{n}\right)=\left(x_{n-d_{k}+1}, \ldots, x_{n}\right)$,

- $P^{\prime}: \mathbb{R}^{n} \rightarrow \mathbb{R}^{n-d_{k}}, P^{\prime}\left(x_{1}, \ldots, x_{n}\right)=\left(x_{1}, \ldots, x_{n-d_{k}}\right)$,

- $Q: \mathbb{R}^{m} \rightarrow \mathbb{R}^{d_{k}}, Q\left(x_{1}, \ldots, x_{m}\right)=\left(x_{n-d_{k}+1}, \ldots, x_{n}\right)$,

- $Q^{\prime}: \mathbb{R}^{m} \rightarrow \mathbb{R}^{m-d_{k}}, Q^{\prime}\left(x_{1}, \ldots, x_{m}\right)=\left(x_{1}, \ldots, x_{n-d_{k}}, x_{n+1}, \ldots, x_{m}\right)$.

Another trivial but useful remark is that we could replace the Euclidean unit ball in the theorem by any convex body, or more generally by any compact $K$ which is an image of $B(0,1)$ under some bilipschitz mapping $\Phi$. Indeed, $\Phi$ induces a natural isomorphism of $\mathscr{C}\left(B(0,1), \mathbb{R}^{m}\right)$ onto $\mathscr{C}\left(K, \mathbb{R}^{m}\right)$ which "commutes" with the map $f \rightarrow M_{k}(f)$ and leaves $f\left(M_{k}(f)\right)$ invariant.

Proposition 1. Let $B\left(x^{i}, R_{i}\right) \subset \mathbb{R}^{n}, i=1, \ldots, k$, be mutually disjoint balls, $f: B \rightarrow \mathbb{R}^{m}$ continuous where $B=\bigcup_{i=1}^{k} B\left(x^{i}, R_{i}\right), \alpha_{R} \geq 0$, and $y \in \mathbb{R}^{m}$. Then there is a continuous map $g: B \rightarrow \mathbb{R}^{m}$ such that

$$
\|f-g\|_{\infty} \leq \max _{x \in B}|f(x)-y|+R_{1}, \quad \text { and } \quad g(B) \subset B\left(y, R_{1}\right)
$$

and that for all $h: B \rightarrow \mathbb{R}^{m}$ continuous with $\|h-g\|_{\infty} \leq R_{1} / 48 k^{3}$ and for all $z \in \mathbb{R}^{d_{k}}$ fulfilling $|z| \leq R_{1} / 2 k$ there are points $\tilde{x}^{i}, \tilde{x}^{i} \in B\left(x^{i}, R_{i}\right)$ satisfying

$$
P\left(\tilde{x}^{1}-x^{1}\right)=Q\left(h\left(\tilde{\tilde{x}}^{1}\right)-y\right)=z
$$

and

$$
h\left(\tilde{x}^{i}\right)=h\left(\tilde{x}^{j}\right), \quad h\left(\tilde{\tilde{x}}^{i}\right)=h\left(\tilde{\tilde{x}}^{j}\right) \quad \text { if } 1 \leq i, j \leq k .
$$

Proof. First of all, using a "nice" substitution of variables which maps the balls $B\left(x^{i}, R_{i}\right), i \geq 2$, onto disjoint balls $B\left(\bar{x}^{i}, R_{1}\right)$ and leaves the conclusion unchanged, we infer that we can assume all the balls to be of the same radius $R\left(=R_{1}\right)$.

We define the following linear mappings from $\mathbb{R}^{n}$ to $\mathbb{R}^{m}$ :

$$
F_{1}\left(x_{1}, \ldots, x_{n}\right)=-\left(x_{1}, \ldots, x_{n}, 0, \ldots, 0\right)
$$

and for $i=2, \ldots, k$

$$
\left[F_{i}\left(x_{1}, \ldots, x_{n}\right)\right]_{l}= \begin{cases}x_{l} & \text { if } 1 \leq l \leq(i-2)(m-n), \\ 0 & \text { if }(i-2)(m-n)<l \leq(i-1)(m-n), \\ x_{l-(m-n)} & \text { if }(i-1)(m-n)<l \leq m\end{cases}
$$


Now, we put $g(x)=y+F_{i}\left(x-x^{i}\right)$ for $x \in B\left(x^{i}, R\right)$. Obviously, (1) is fulfilled. To prove the statements concerning $h$, we introduce two auxiliary mappings from $B\left(x^{1}, R\right) \times \cdots \times B\left(x^{k}, R\right) \subset\left(\mathbb{R}^{n}\right)^{k}$ into $\left(\mathbb{R}^{m}\right)^{(k-1)} \times \mathbb{R}^{d_{k}} \cong \mathbb{R}^{n^{*} k}$ :

$\phi_{P}^{h}\left(\bar{x}^{1}, \ldots, \bar{x}^{k}\right)=\left(h\left(\bar{x}^{2}\right)-h\left(\bar{x}^{1}\right), h\left(\bar{x}^{3}\right)-h\left(\bar{x}^{1}\right), \ldots, h\left(\bar{x}^{k}\right)-h\left(\bar{x}^{1}\right), P\left(\bar{x}^{1}-x^{1}\right)\right)$

and

$$
\begin{aligned}
& \phi_{Q}^{h}\left(\bar{x}^{1}, \ldots, \bar{x}^{k}\right) \\
& \quad=\left(h\left(\bar{x}^{2}\right)-h\left(\bar{x}^{1}\right), h\left(\bar{x}^{3}\right)-h\left(\bar{x}^{1}\right), \ldots, h\left(\bar{x}^{k}\right)-h\left(\bar{x}^{1}\right), Q\left(h\left(\bar{x}^{1}\right)-y\right)\right) .
\end{aligned}
$$

First, we consider $\Phi_{P}=\Phi_{P}^{g}$ and $\Phi_{Q}=\Phi_{Q}^{g}$. Note that $\Phi_{Q}=\Phi_{P}(=\Phi)$ due to the choice of $F_{1}$. Moreover, the map

$$
\left(\Delta^{1}, \ldots, \Delta^{k}\right) \rightarrow \Phi\left(x^{1}+\Delta^{1}, \ldots, x^{n}+\Delta^{n}\right)
$$

is a linear isomorphism restricted to $(B(0, R))^{k}$. Indeed, one easily verifies that the equation

$$
\left(F_{2}\left(\Delta^{2}\right)-F_{1}\left(\Delta^{1}\right), \ldots, F_{k}\left(\Delta^{k}\right)-F_{1}\left(\Delta^{1}\right), P\left(\Delta^{1}\right)\right)=z \in \mathbb{R}^{n \cdot k}
$$

has the following unique solution:

$$
\Delta_{j}^{1}= \begin{cases}z_{n(k-1)+j} & \text { for } j=(k-1)(m-n)+1, \ldots, n, \\ z_{l \cdot m+j} & \text { for } 1 \leq l<k \text { and }(l-1)(m-n)<j \leq l(m-n),\end{cases}
$$

and for $l=1, \ldots, k-1$

$$
\Delta_{j}^{l+1}= \begin{cases}z_{l \cdot m+j}-\Delta_{j}^{1} & \text { for } 1 \leq j \leq(l-1)(m-n), \\ z_{l \cdot m+j+(m-n)}-\Delta_{j+(m-n)}^{1} & \text { for }(l-1)(m-n)<j \leq 2 n-m, \\ z_{l \cdot m+j+(m-n)} & \text { for } 2 n-m<j \leq n .\end{cases}
$$

(We recall that $(k-2)(m-n)=2 n-m-d_{k} \leq 2 n-m$.) In particular, we have for this solution $\left|\Delta^{1}\right| \leq|z|$ and $\left|\Delta^{j}\right| \leq\left|\Delta^{1}\right|+|z| \leq 2|z|$ if $j \geq 2$. So we obtain for any $\Delta^{i} \in B(0, R)$ the estimate

$$
\frac{\sum_{i=1}^{k}\left|\Delta^{i}\right|}{2 k-1} \leq\left|\Phi\left(x^{1}+\Delta^{1}, \ldots, x^{n}+\Delta^{n}\right)\right| .
$$

Hence, $|\Phi(z)| \geq R /(2 k-1)$ for any boundary point $z$ of the domain $U\left(x^{1}, R\right)$ $\times \cdots \times U\left(x^{n}, R\right)$. Since also $\left\|\Phi_{P}^{h}-\Phi_{P}^{h^{\prime}}\right\|_{\infty},\left\|\Phi_{Q}^{h}-\Phi_{Q}^{h^{\prime}}\right\|_{\infty} \leq 2 k\left\|h-h^{\prime}\right\|_{\infty}$, we see that for any $h \in U\left(g, R /\left(8 k^{3}\right)\right)$, any $t \in[0,1]$, and any

$$
z \in \partial\left(B\left(x^{1}, R\right) \times \cdots \times B\left(x^{n}, R\right)\right)
$$

the inequalities

$$
\left|\Phi_{P}^{h_{t}}(z)\right|,\left|\Phi_{Q}^{h_{t}}(z)\right| \geq \frac{R}{2 k-1}-2 k \frac{R}{8 k^{3}}>\frac{R}{2 k}
$$

holds with $h_{t}=g+t(h-g)$. Therefore, the classical degree theory for continuous mappings, see e.g. [6, Theorem 3.1(d3)] ensures that, for each point $z \in \mathbb{R}^{n \cdot k}$ with $|z|<R /(2 k)$,

$$
\operatorname{deg}\left(\Phi_{P}^{h}, z\right)=\operatorname{deg}\left(\Phi_{Q}^{h}, z\right)=\operatorname{deg}(\Phi, z)=\operatorname{sign}(\operatorname{det}(D \Phi)) \neq 0
$$


holds. In particular, the point $\left(0, \ldots, 0, z_{1}, \ldots, z_{d_{k}}\right) \in\left(\mathbb{R}^{m}\right)^{(k-1)} \times \mathbb{R}^{d_{k}}$ belongs to both sets

$$
\Phi_{P}^{h}\left(B\left(x^{1}, R\right) \times \cdots \times B\left(x^{k}, R\right)\right) \quad \text { and } \Phi_{Q}^{h}\left(B\left(x^{1}, R\right) \times \cdots \times B\left(x^{k}, R\right)\right)
$$

whenever $|z| \leq R /(2 k)$. But from this statement and the definition of the maps $\Phi_{P}^{h}, \Phi_{Q}^{h}$ the conclusion of our proposition follows immediately.

Corollary 1. Let $B_{i} \subset \mathbb{R}^{n}, i=1, \ldots, k$, be mutually disjoint balls, $f: B \rightarrow \mathbb{R}^{m}$ continuous where $B=\bigcup_{i=1}^{k} B_{i}$ and $y \in \mathbb{R}^{m}$, and $R>0$ be given. Then there is a continuous map $g: B \rightarrow \mathbb{R}^{m}$ such that

$$
\|f-g\|_{\infty} \leq \max _{x \in B}|f(x)-y|+R, \quad g(B) \subset B(y, R),
$$

and that for all $h: B \rightarrow \mathbb{R}^{m}$ continuous with $\|h-g\|_{\infty} \leq R / 48 k^{3}$ and for all $z \in \mathbb{R}^{d_{k}}$ fulfilling $|z| \leq R / 2 k$ there are points $\tilde{x}^{i} \in B_{i}$ satisfying

$$
Q\left(h\left(\tilde{x}^{1}\right)-y\right)=z \text { and } h\left(\tilde{x}^{i}\right)=h\left(\tilde{x}^{j}\right), \quad \text { for } 1 \leq i, j \leq k .
$$

Proof. Follows immediately from Proposition 1, e.g. using a suitable diffeomorphism of $\mathbb{R}^{n}$ which maps the $B_{1}, \ldots, B_{k}$ onto some disjoint balls of radius $R$.

Note that Proposition 1 and the fact that $d_{k}<n$ immediately imply that for any nonvoid $U \subset[0,1]^{n}$ open and for any constant $K$ the set of all $f$ fulfilling $\mathscr{H}^{d_{k}}\left(M_{k}(f) \cap U\right)>K$ and $\mathscr{H}^{d_{k}}\left(f\left(M_{k}(f) \cap U\right)\right)>K$ has a dense interior in $\mathscr{C}\left([0,1]^{n}, \mathbb{R}^{m}\right)$. Hence, we see that for typical $f$ both $M_{k}(f)$ and $f\left(M_{k}(f)\right)$

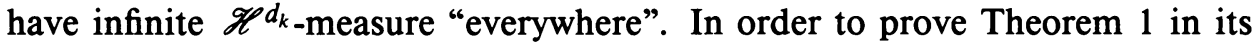
whole strength, we have to play the Banach-Mazur game with a strategy which is based on the just-proved proposition and corollary and which builds trees leading to perfect sets inside sufficiently many slices of the form $M_{k}(f) \cap P^{-1}(t)$, $f\left(M_{k}(f)\right) \cap Q^{-1}(t)$. This is motivated by the following simple observation.

Lemma 1. Let $M \subset \mathbb{R}^{i}$ be arbitrary and $f: \mathbb{R}^{i} \rightarrow \mathbb{R}^{j}$ be lipschitz. If

$$
\mathscr{H}^{l}\left(\left\{t ; f^{-1}(t) \cap M \text { is uncountable }\right\}\right)>0,
$$

then $M$ is not of $\sigma$-finite l-dimensional Hausdorff measure.

Proof. On the contrary, suppose $M \subset \bigcup_{p=1}^{\infty} M_{p}$ and $\mathscr{H}^{l}\left(M_{p}\right)<\infty$ for all $p$. Due to [7, Theorem 2.10.25] we have the estimate

$$
r \mathscr{H}^{l}\left\{t ; \operatorname{card}\left(f^{-1}(t) \cap M_{p}\right) \geq \gamma\right\} \leq(\operatorname{lip} f)^{l} \mathscr{H}^{n}\left(M_{p}\right) \text { for all } r
$$

which implies that for $\mathscr{H}^{l}$-almost every $t$ and all $p \geq 1$ the set $f^{-1}(t) \cap M_{p}$ is countable. But then also $f^{-1}(t) \cap M$ is countable for almost each $t$.

Now we are ready to prove

Proposition 2. If $k \geq 2$, then for typical continuous $f: B\left(0,1 ; \mathbb{R}^{n}\right) \rightarrow \mathbb{R}^{m}$ is

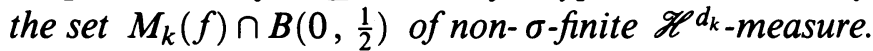

Proof. During this proof we will use the following notation. Whenever $B=$ $B(x, r)$ is a ball and $\alpha \geq 0$ then $\alpha \cdot B=B(x, \alpha r)$.

As already mentioned, we play the Banach-Mazur game. In the first step, let us be given the first answer $U\left(f_{1}, \varepsilon_{1}\right)$ of Player A. We choose an $R_{1}^{\prime} \in$ 
$\left(0, \frac{1}{4}\right)$ such that $\left|f_{1}(x)-f_{1}\left(x^{\prime}\right)\right|<\varepsilon_{1} / 2$ whenever $\left|x-x^{\prime}\right| \leq R_{1}^{\prime}$, and then we select $R_{1} \in\left(0, \min \left\{R_{1}^{\prime}, \frac{\varepsilon_{1}}{2}\right\}\right)$ and points $x^{1}, \ldots, x^{k}$ such that the balls $B\left(x^{i}, 2 R_{1}\right) \subset B\left(0, R_{1}^{\prime}\right)$ are mutually disjoint. Further, we put $y=f_{1}(0)$. According to Proposition 1 we find a map $f_{2} \in U\left(f, \frac{\varepsilon_{1}}{2}+R_{1}\right)$ and $\varepsilon_{2} \in\left(0, \varepsilon_{1}-\right.$ $\left.\left\|f_{1}-f_{2}\right\|_{\infty}\right)$ such that for any $h \in U\left(f_{2}, \varepsilon_{2}\right)$ and any $z \in B\left(0, \frac{R_{1}}{2 k} ; \mathbb{R}^{d_{k}}\right)$ there exist points $\tilde{x}^{i} \in B\left(x^{i}, R_{1}\right), i=1, \ldots, k$, with $P\left(\tilde{x}^{1}-x^{1}\right)=z$ and $h\left(\tilde{x}^{i}\right)=$ $h\left(\tilde{x}^{1}\right)$. These will form the roots of our trees, so we introduce the following, more appropriate notation: $S=B\left(P\left(x^{1}\right), R_{1} / 2 k ; \mathbb{R}^{d_{k}}\right), x[\varnothing, \varnothing, i]=x^{i}$, and $B[\varnothing, \varnothing, i]=B\left(x[\varnothing, \varnothing, i], R_{1}\right)$ for $i \leq k$. Then we return the open ball $U\left(f_{2}, \varepsilon_{2}\right)$.

In the second step, given A's answer $U\left(f_{3}, \varepsilon_{3}\right)$, we fix an $R_{2}$ in the interval $\left(0, R_{1} /(200 k)\right)$ such that $\left|f_{3}(x)-f_{3}\left(x^{\prime}\right)\right|<\varepsilon_{3} / 8$ whenever $\left|x-x^{\prime}\right| \leq 200 k R_{2}$. Now let $D_{1} \subset B\left(0, R_{1} / 2 k ; \mathbb{R}^{d_{k}}\right)$ be a set maximal among all subsets $D$ of this ball with the property $\left|t-t^{\prime}\right| \geq R_{2} /(2 k)$ for all different $t, t \in D$. (A simple volume estimate shows that card $D_{1} \leq\left(\frac{2 R_{1}}{R_{2}}+1\right)^{k}$, but we need only the obvious fact that $D_{1}$ is finite.) We claim that for all $i=1, \ldots, k, j=0,1$, and $t \in D_{1}$ there are closed balls $B[(j),(t), i]$ with centers $x[(j),(t), i]$ such that all the $B[(j),(t), 1]$ have radius $R_{2}$ and that

$$
\begin{gathered}
P\left(B\left(x[\varnothing, \varnothing, 1], \frac{R_{1}}{2 k}\right)\right) \\
\subset \bigcup_{t \in D_{1}} P\left(B\left(x[(j),(t), 1], \frac{R_{2}}{2 k}\right)\right) \quad \text { for } j=0,1, \\
P(x[(0),(t), 1])=P(x[(1),(t), 1])=t \text { for all } t \in D_{1}, \\
\left|f_{3}(x[(j),(t), i])-f_{3}(x[(j),(t), 1])\right|<\frac{\varepsilon_{3}}{4} \text { for all } i, j, t, \\
2 B[(j),(t), i] \cap 2 B\left[\left(j^{\prime}\right),\left(t^{\prime}\right), i\right]=\varnothing \quad \text { whenever }(j, t) \neq\left(j^{\prime}, t^{\prime}\right), \\
2 B[(j),(t), i] \subset 2 B[\varnothing, \varnothing, i] \text { for all } i, j, t .
\end{gathered}
$$

For this purpose we choose a linear order $\prec$ of the set $D_{1}$ and suppose that for some $t \in D_{1}$ the $x\left[(j),\left(t^{\prime}\right), i\right] \subset B\left(x^{i}, R_{1}\right)$ already chosen for all $i, j, t^{\prime} \prec$ $t$, are different whenever their indices differ and fulfill (3) as well as (5), for $i=1$. According to the choice of our answer $U\left(f_{2}, \varepsilon_{2}\right)$ we find points $\bar{x}^{i} \in$ $B[\varnothing, \varnothing, i]$, with $f_{3}\left(\bar{x}^{i}\right)=f_{3}\left(\bar{x}^{1}\right)$ for all $i \leq k$ and $P\left(\bar{x}^{1}-x[\varnothing, \varnothing, 1]\right)=t$. First, we consider the system $\mathscr{F}$ of all balls $B\left(x\left[(j),\left(t^{\prime}\right), 1\right], 4 R_{2}\right), j=0,1$ and $t^{\prime} \prec t$, which satisfy $t \in P(B)$. Since all of the $P$-projections of balls in $\mathscr{F}$ are centered in $D_{1}$ and at most two of them in the same point, a straightforward volume estimate shows that $N=$ card $\mathscr{F}$ fulfills

$$
\frac{N}{2}\left(\frac{R_{2}}{4 k}\right)^{d_{k}} \leq\left(4 R_{2}+\frac{R_{2}}{4 k}\right)^{d_{k}}
$$

and, therefore, $N \leq 2(16 k+1)^{d_{k}} \leq(40 k)^{d_{k}}$. Next, we look at the ball $B^{\prime}=$ $P^{\prime}\left(B\left(\bar{x}^{1}, 165 k R_{2}\right)\right) \subset \mathbb{R}^{n-d_{k}}$ and the set $M=B^{\prime} \backslash \bigcup_{B \in \mathscr{F}} P^{\prime}(B)$. We compute that $|M| \geq((N+1)-N)\left|B\left(0,4 R_{2} ; \mathbb{R}^{n-d_{k}}\right)\right|>\left|B\left(0,2 R_{2} ; \mathbb{R}^{n-d_{k}}\right)\right|$, here $|\cdot|$ being the Lebesgue measure on $\mathbb{R}^{n-d_{k}}$. Hence, the isodiametric inequality (see 
e.g. [4]) ensures the existence of two points $z^{0}, z^{1} \in M$ with $\left|z^{0}-z^{1}\right|>4 R_{2}$. The desired two points are now defined by the conditions

$$
P(x[(j),(t), 1]-x[\varnothing, \varnothing, 1])=t \quad \text { and } \quad P^{\prime}(x[(j),(t), 1])=z^{j} .
$$

It follows from our definition of $M$ that the balls $B[(j),(t), 1]$ again satisfy (3) and (5) (together with the other $\left.B\left[\left(j^{\prime}\right),\left(t^{\prime}\right), 1\right], t^{\prime} \prec t\right)$. Now we choose the points $x[(j),(t), i] \in B[\varnothing, \varnothing, i] \cap B\left(\bar{x}^{i}, R_{2}\right), j=0,1, i \geq 2$, in such a way that all the points chosen by now will be different. Note that this implies $\left|f_{3}(x[(j),(t), i])-f_{3}\left(\bar{x}^{i}\right)\right|<\varepsilon_{3} / 8$ due to our choice of $R_{2}$, which yields (4). If we have proceeded the whole set $D_{1}$ in this manner, we simply choose all the $B[(j),(t), i], i \geq 2$, sufficiently small to ensure (5), (6). Finally, the maximality of $D_{1}$ guarantees (2). (Note that all these considerations can be omitted in case $d_{k}=0$ where $S=\{0\}=D_{1}$ and all we have to choose are two appropriate "disjoint" subballs in each $B[(j), 0, i])$. Now we can apply Proposition 1 to each of the systems $\{B[(j),(t), i] ; i \leq k\}$ for $j=0,1$ and $t \in D_{1}$. So we obtain a mapping $f_{4} \in U\left(f_{3}, \varepsilon_{3}\right)$ and an $\varepsilon_{4} \in\left(0, \varepsilon_{3}-\right.$ $\left.\left\|f_{3}-f_{4}\right\|_{\infty}\right)$ such that for all $h \in U\left(f_{4}, \varepsilon_{4}\right)$, for any $j=0,1, t \in D_{1}$, and for any $z \in B\left(0, R_{2} /(2 k) ; \mathbb{R}^{d_{k}}\right)$ there are $\bar{x}^{i} \in B[(j),(t), i]$ fulfilling $P\left(\bar{x}^{1}-x[(j),(t), 1]\right)=z$ and $h\left(\bar{x}^{i}\right)=h\left(\bar{x}^{1}\right)$ for all $i \leq k$.

We return to $U\left(f_{4}, \varepsilon_{4}\right)$. According to the foregoing statement about $h$, after receiving the answer $U\left(f_{5}, \varepsilon_{5}\right)$ we can repeat the construction of Step II for each of $j=0,1$ and $t \in D_{1}$ with the family $\{B[(j),(t), i] ; i \leq k\}$ instead of $\{B[(\varnothing, \varnothing, i] ; i \leq k\}$. Iterating this procedure, we are always in a position to obtain a play $\left\{U\left(f_{l}, \varepsilon_{l}\right)\right\}_{l=1}^{\infty}$ of the Banach-Mazur game, sequences (in $l=1,2, \ldots$ ) of

- $R_{l}$ with $0<R_{l+1}<R_{l} /(200 k)$,

- $D_{l} \subset B\left(0, R_{l} /(2 k) ; \mathbb{R}^{d_{k}}\right)$ maximal among all subsets of this ball having minimal distance between its members at least $R_{l+1} /(2 k)$, and $k$ trees (with height $l$ of the corners running from 0 to $\infty$ ),

- of balls $B[\omega, \sigma, i]$ centered in $x[\omega, \sigma, i]$ for $i=1, \ldots, k, \omega \in$ $\{0,1\}^{l}$, and $\sigma \in D_{1} \times \cdots \times D_{l}$ and of radii $R_{l+1}$ provided $i=1$,

which all together satisfy for any $i \leq k, l \geq 1, \sigma \in D_{1} \times \cdots \times D_{l}, \omega \in$ $\{0,1\}^{l}$, and $t, t^{\prime} \in D_{l+1}, j, j^{\prime}=0,1$ with $(j, t) \neq\left(j^{\prime}, t^{\prime}\right)$ the following five conditions:

$$
\begin{gathered}
2 B[\omega j, \sigma t, i] \subset 2 B[\omega, \sigma, i], \\
2 B[\omega j, \sigma t, i] \cap 2 B\left[\omega j^{\prime}, \sigma t^{\prime}, i\right]=\varnothing, \\
P\left(\frac{1}{2 k} B[\omega, \sigma, 1]\right) \subset \bigcup_{\bar{t} \in D_{l+1}} P\left(\frac{1}{2 k} B[\omega j, \sigma \bar{t}, 1]\right), \\
P(x[\omega j, \sigma t, 1]-x[\omega, \sigma, 1])=t
\end{gathered}
$$

and finally

$$
P\left(\frac{1}{2 k} B[\omega, \sigma, 1]\right) \subset P\left(B[\omega, \sigma, 1] \cap \bigcap_{i^{\prime}=2}^{k} f_{2 l+2}^{-1}\left(f_{2 l+2}\left(B\left[\omega, \sigma, i^{\prime}\right]\right)\right)\right)
$$


(These conditions are not independent; e.g. (10) is helpful during the construction, but at the end we need only its consequence (9).)

Let $f$ be the uniform limit of the $f_{l}$. We are done if we show that $M_{k}(f) \cap$

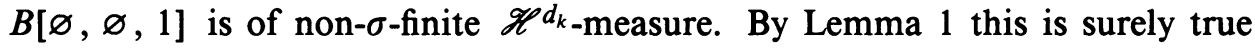
once we know that for any $t \in S$ the set

$$
C=M_{k}(f) \cap B[\varnothing, \varnothing, 1] \cap P^{-1}(t)
$$

contains a perfect subset. But indeed, for any such $t$ there is due to (9) a sequence $\left\{t_{l}\right\}_{l=1}^{\infty} \in \prod_{l=1}^{\infty} D_{l}$ such that for $l \geq 1, \sigma \in\{0,1\}^{l}$,

$$
t \in P\left(\frac{1}{2 k} B\left[\omega,\left(t_{1}, \ldots, t_{l}\right), 1\right]\right) .
$$

If we denote, for $\omega \in\{0,1\}^{l}, B^{\omega}=B\left[\omega,\left(t_{1}, \ldots, t_{l}\right), 1\right]$, we infer from (11) the existence of some

$$
x^{\omega} \in B^{\omega} \cap P^{-1}(t) \cap \bigcap_{i=2}^{k} f_{l+1}^{-1}\left(f_{l+1}(B[\varnothing, \varnothing, i])\right) .
$$

Using the uniform convergence of the $f_{l}$ 's one sees easily that also, for any cluster point $x$ of $\left\{x^{\omega} ; \omega\right\}$ and any $i=1, \ldots, k, f^{-1}(f(x)) \cap B[\varnothing, \varnothing, i] \neq \varnothing$ holds; therefore $x \in M_{k}(f)$. Moreover $R_{l} \searrow 0$ and (7), (8) imply that the set of all cluster points of $\left\{x^{\omega} ; \omega\right\}$ has no isolated point. Consequently, it is a perfect subset of $C$.

Proposition 3. For typical $f: C\left(0,1, \mathbb{R}^{n}\right) \rightarrow \mathbb{R}^{m}$ the set $f\left(M_{k}(f) \cap B(0,1 / 2)\right)$ is of non- $\sigma$-finite $\mathscr{H}^{d_{k} \text {-measure. }}$

Proof. We mimic the proof of Proposition 2, obtain a play $\left\{U\left(f_{j}, \varepsilon_{j}\right)\right\}_{j=1}^{\infty}$ of the Banach-Mazur game and construct simultaneously the following objects:

- mutually disjoint balls $B_{1}, \ldots, B_{k} \subset B\left(0,1 / 2, \mathbb{R}^{n}\right)$,

- a sequence $\left\{R_{l}\right\}_{l=1}^{\infty}$ with $0<R_{l+1}<R_{l} / 4$,

- a sequence $\left\{D_{l}\right\}_{l=1}^{\infty}$ of sets maximal among all subsets of $B\left(0, R_{l} /(2 k)\right.$; $\mathbb{R}^{d_{k}}$ ), having minimal distance between its members at least $R_{l+1} /(2 k)$,

- a tree of points $y[\omega, \sigma] \in \mathbb{R}^{m}$ where $\omega \in\{0,1\}^{l}, \sigma \in D_{1} \times \cdots \times D_{l}$, and $l=0,1, \ldots$,

which all together satisfy for any $l \geq 0, \sigma \in D_{1} \times \cdots \times D_{l}, \omega \in\{0,1\}^{l}$, and $t, t^{\prime} \in D_{l+1}, j, j^{\prime}=0,1$ with $(j, t) \neq\left(j^{\prime}, t^{\prime}\right)$ the following five conditions:

$$
\begin{gathered}
B\left(y[\omega j, \sigma t], 2 R_{l+2}\right) \subset B\left(y[\omega, \sigma], 2 R_{l+1}\right), \\
B\left(y[\omega j, \sigma t], 2 R_{l+2}\right) \cap B\left(y\left[\omega j^{\prime}, \sigma t^{\prime}\right], 2 R_{l+2}\right)=\varnothing, \\
Q\left(B\left(y[\omega, \sigma], \frac{R_{l+1}}{2 k}\right)\right) \subset \bigcup_{t \in D_{l+1}} Q\left(B\left(y[\omega j, \sigma t], \frac{R_{l+2}}{2 k}\right)\right), \\
Q(y[\omega j, \sigma t]-y[\omega, \sigma])=t
\end{gathered}
$$

and finally

$$
Q\left(B\left(y[\omega, \sigma], \frac{R_{l+1}}{2 k}\right)\right) \subset Q\left(B\left(y[\omega, \sigma], R_{l+1}\right) \cap \bigcap_{i=1}^{k} f_{l+2}\left(\operatorname{int}\left(B^{i}\right)\right)\right) .
$$


From this we can as in the proof of Proposition 2 conclude that for any uniform limit $f$ of the $f_{j}^{\prime}$ 's and $t \in Q\left(B\left(y[\varnothing, \varnothing], \frac{R_{1}}{2 k}\right)\right)$ the set $Q^{-1}(t) \cap \bigcap_{i=1}^{k} f\left(B^{1}\right)$ contains a perfect subset. Since this means that we won the game, our proof is finished.

Next, we derive the result used for the upper estimate of the Hausdorff dimension of the sets $M_{k}$.

Lemma 2. The set $U$ of all

$$
\left(x_{0}^{1}, \ldots, x_{n}^{1}, x_{0}^{2}, \ldots, x_{n}^{2}, \ldots, x_{0}^{k}, \ldots, x_{n}^{k}\right) \in\left(\mathbb{R}^{m}\right)^{(n+1) k}
$$

for which

$$
d=\operatorname{dim}\left(\bigcap_{i=1}^{k} \operatorname{AS}\left(x_{0}^{i}, \ldots, x_{n}^{i}\right)\right) \leq d_{k}
$$

holds has an interior dense in $X=\mathbb{R}^{m(n+1) k}$. Here $\operatorname{dim} \varnothing=-\infty$, and we denote by $\operatorname{AS}\left(x_{0}, \ldots, x_{n}\right)$ the smallest affine subspace of $\mathbb{R}^{m}$ containing $\left\{x_{0}, \ldots, x_{n}\right\}$. (We will simply write AS if the $x_{0}, \ldots, x_{n}$ follow from the context.)

Proof. We also define for $x_{0}, \ldots, x_{n} \in \mathbb{R}^{n}$

$$
\mathbf{L S}=\mathbf{L S}\left(x_{0}, \ldots, x_{n}\right)=\operatorname{span}\left(\left\{x_{j}-x_{0} ; 1 \leq j \leq n\right\}\right)
$$

and, for $\bar{x} \in X, \operatorname{LS}(\bar{x})=\bigcap_{i=1} \operatorname{LS}\left(x_{0}^{i}, \ldots, x_{n}^{i}\right), \bar{d}(\bar{x})=\operatorname{dim} \operatorname{LS}(\bar{x})$. Similarly we have $\operatorname{AS}(\bar{x})$ and $d(\bar{x})$.

Since $\mathrm{AS}=y+\mathrm{LS}$ for any $y \in \mathrm{AS}$, we infer that always $\bar{d}(\bar{x}) \geq d(\bar{x})$. If we consider the first case $d_{k} \geq 0$, it suffices to show that $\bar{d}(\bar{x}) \leq d_{k}$ on some dense open subset $U^{\prime}$ of $X$. First, note that for all $l \geq 1$ the set

$$
U_{l}=\left\{\left(x_{1}, \ldots, x_{n}\right) ; \operatorname{dim} \operatorname{span}\left(\left\{x_{1}, \ldots, x_{n}\right\}\right) \geq l\right\}
$$

is open in $\left(\mathbb{R}^{m}\right)^{n}$; this immediately follows from the consideration of appropriate subdeterminants. Moreover, if $l \leq n$ then obviously this set is also dense. Consequently, the set $X_{r}$ of all $\bar{x} \in X$ such that $\operatorname{dim}\left(\operatorname{LS}\left(x_{0}^{i}, \ldots, x_{0}^{i}\right)\right)=n$ for all $i \leq k$ is a dense open subset of $X$. Furthermore, it is clear that for $y^{1}, \ldots, y^{\bar{k}} \in \mathbb{R}^{m}, \bar{x} \in X_{r}$ then condition $y^{i} \in \operatorname{LS}\left(x_{0}^{i}, \ldots, x_{n}^{i}\right)$ for all $i$ holds iff $\operatorname{dim}\left(\operatorname{span}\left(\left\{y, x_{1}^{i}-x_{0}^{i}, \ldots, x_{n}^{i}-x_{0}^{i}\right\}\right)\right) \leq n$. Hence, the set

$$
\left\{\left(\bar{x}, y^{1}, \ldots, y^{k}\right) ; y^{i} \in \operatorname{LS}\left(\bar{x}_{0}^{i}, \ldots, \bar{x}_{n}^{i}\right) \text { for all } i \leq k\right\}
$$

is closed in $X_{r} \times\left(\mathbb{R}^{m}\right)^{k}$. In particular, the set $\{(\bar{x}, y) ; y \in \operatorname{LS}(\bar{x})\}$ is closed in $X_{r} \times \mathbb{R}^{m}$. Using this fact and the compactness of the family of all " $l$ dimensional" orthogonal systems, one easily sees that for any $l \geq 0$ $\left\{\bar{x} \in X_{r} ; \bar{d}(\bar{x}) \geq l\right\}$ is closed in $X_{r}$. So, we are done if we can show that $\left\{\bar{x} \in X_{r} ; \bar{d}(\bar{x}) \leq d_{k}\right\}$ is dense in $X_{r}$. The proof of this fact is based on induction with respect to $k$ and the well-known formula

$$
\operatorname{dim}(U \cap V)=\operatorname{dim}(U)+\operatorname{dim}(V)-\operatorname{dim}(\operatorname{span}(U \cup V))
$$

valid for all linear subspaces $U, V$ of $\mathbb{R}^{m}$. The induction step follows easily from a simple perturbation argument saying that for any linear subspace $U$ of $\mathbb{R}^{m}$ and any $x_{0}, \ldots, x_{d} \in \mathbb{R}^{m}$ where $d+\operatorname{dim}(U) \leq m$ and any $\varepsilon>0$ there are $y_{i} \in B\left(x_{i}, \varepsilon\right), i=0, \ldots, d$, such that $y_{i}-y_{0} \notin \operatorname{span}\left(U \cup\left\{y_{j}-y_{0} ; 1 \leq j<i\right\}\right)$ whenever $1 \leq i \leq d$. 
Finally, we have to consider the case $d_{k}<0$; obviously it suffices to restrict to the case $d_{k} \geq n-m$. This time we use the fact that those points in $X_{r}$ which satisfy $\bar{d}(\bar{x}) \geq 1$ or $\operatorname{AS}(\bar{x})=\varnothing$ form a closed subset $X^{\prime}$. Indeed, let $\bar{x}_{p} \rightarrow \bar{x} \in X_{r}$ and all $\bar{x}_{p} \in X^{\prime}$. We may assume the existence of a $y_{p} \in \operatorname{AS}\left(\bar{x}_{p}\right)$ for all $p$; otherwise $\bar{d}(\bar{x}) \geq 1$. Hence, $y_{p}-\left(\bar{x}_{p}\right)_{0}^{i} \in \operatorname{LS}\left(\left(\bar{x}_{p}\right)_{0}^{i}, \ldots,\left(\bar{x}_{p}\right)_{n}^{i}\right)$ for all $i, p$. If $\left|y_{p}\right| \rightarrow \infty$, then any cluster point $\bar{y}$ of the sequence $y_{p} /\left|y_{p}\right|$ is also a cluster point of $\left\{\left(y_{p}-\left(\bar{x}_{p}\right)_{0}^{i}\right) /\left|y_{p}\right|\right\}$ for any $i \leq k$. Therefore, it belongs to $\operatorname{LS}(\bar{x})$, showing that $\bar{d}(\bar{x}) \geq 1$. Otherwise, we find a cluster point $\bar{y}$ of the sequence $\left\{y_{p}\right\}$ itself. We conclude that $\bar{y}-\bar{x}_{0}^{i} \in \operatorname{LS}\left(\bar{x}_{0}^{i}, \ldots, \bar{x}_{n}^{i}\right)$ for any $i \leq k$, which implies $\bar{y} \in \operatorname{AS}(\bar{x})$. Summarizing, $\bar{x} \in X^{\prime}$ in both cases. The density of the open set $X_{r} \backslash X^{\prime}$ is now a consequence of the first case for $m-n+d_{k} \leq 0$, of the already stated perturbation argument, and of the following observation. If $U \subset \mathbb{R}^{m}$ is linear and $x_{0}, \ldots, x_{p} \in \mathbb{R}^{m}$ fulfill $x_{j} \notin \operatorname{span}\left(\left\{x^{i} ; i<j\right\} \cup U\right)$ for all $j=0, \ldots, p$, then $U \cap \operatorname{AS}\left(x_{0}, \ldots, x_{p}\right)=\varnothing$. Indeed, otherwise we could fix $y \in U$ and $\lambda_{1}, \ldots, \lambda_{p} \in \mathbb{R}$ with $y-x_{0}=\sum_{i=1}^{p} \lambda_{i}\left(x_{i}-x_{0}\right)$. This implies $0=-y-\left(\sum_{i=1}^{p} \lambda_{i}-1\right) x_{0}+\sum_{i=1}^{p} \lambda_{i} x_{i}$; hence $\sum_{i=1}^{p} \lambda_{i}=1$ and $\lambda_{1}=\cdots=\lambda_{p}=0$, a contradiction finishing our proof.

Proposition 4. Let $\varphi$ be a Hausdorff function with $\lim _{r \searrow 0} \varphi(r) / r^{d_{k}}=0$. Then $\mathscr{H}^{\varphi}\left(M_{k}(f)\right)=\mathscr{H}^{\varphi}\left(f\left(M_{k}(f)\right)\right)=0$ holds for typical continuous $f: B\left(0,1 / 2 ; \mathbb{R}^{n}\right)$ $\rightarrow \mathbb{R}^{m}$.

Proof. As already mentioned, we can consider instead of $\mathscr{C}\left(B(0,1), \mathbb{R}^{m}\right)$ also the space $\mathscr{C}=\mathscr{E}\left(S, \mathbb{R}^{m}\right)$ where $S$ is a simplex in $\mathbb{R}^{n}$. We define for any $l \geq 1$ the open sets $\mathscr{G}_{l} \subset \mathscr{C}$ consisting of all functions $f$ for which there exist an $\eta>0$ and a set $M=M(f, l) \subset S$ satisfying

$$
\begin{gathered}
\mathscr{H}_{\infty}^{\varphi}(M)<1 / l \text { and } \mathscr{H}_{\infty}^{\varphi}(U(f(M), \eta))<1 / l, \\
\operatorname{diam}\left(\left\{f\left(x^{j}\right) ; j \leq k\right\}\right)>\eta \text { if some } x^{j} \notin M \text { and } \min _{i<j}\left|x^{i}-x^{j}\right| \geq \frac{1}{l} .
\end{gathered}
$$

Suppose that $f \in \bigcap_{l=1}^{\infty} \mathscr{G}_{l}$. We put $\widetilde{M}=\liminf _{l \rightarrow \infty} M(f, l)$ and claim $M_{k}(f) \subset \widetilde{M}$. Indeed, suppose that $x^{1}, \ldots, x^{k} \in S$ fulfill $x^{1} \notin \widetilde{M}$ and $\min _{i<j}\left|x^{i}-x^{j}\right|>\delta>0$. Then $x^{1} \notin M(f, l)$ for some $l \geq 1 / \delta$, and hence $f\left(x^{1}\right)=\cdots=f\left(x^{k}\right)$ cannot be true. Therefore, we also have $f\left(M_{k}(f)\right) \subset$ $\liminf _{l \rightarrow \infty} f(M(f, l))$ and conclude that $\mathscr{H}_{\infty}^{\varphi}\left(M_{k}(f)\right) \leq \mathscr{H}_{\infty}^{\varphi}\left(f\left(M_{k}(f)\right)\right) \leq$ $\lim _{l \rightarrow \infty} 1 / l=0$.

So all we need to show is that each of the $\mathscr{G}_{l}$ is dense in $\mathscr{C}$. Since

$$
\mathscr{H}_{\infty}^{\varphi}\left([0,1]^{d_{k}} \times\left[-\frac{1}{2 j}, \frac{1}{2 j}\right]\right) \leq \varphi\left(\sqrt{d_{k}+1} / j\right) j^{d_{k}} \searrow 0 \text { as } j \rightarrow \infty,
$$

we see that $f \in \mathscr{G}_{l}$ whenever there is a set $M$ such that $M$ and $f(M)$ are unions of finitely many bounded sets contained in $d_{k}$-dimensional affine spaces and that always $\operatorname{diam}\left(\left\{f\left(x^{j}\right) ; j \leq k\right\}\right)>0$ if $x^{1} \notin M$ and $\min _{i<j}\left|x^{i}-x^{j}\right| \geq \frac{1}{l}$. Indeed, in this case compactness yields for any $\rho>0$ a uniform estimate for this diameter on

$$
(S \backslash U(M, \rho)) \times S \times \cdots \times S \cap\left\{\left(x^{1}, \ldots, x^{n}\right) ; \min _{i<j}\left|s^{i}-x^{j}\right| \geq \frac{1}{l}\right\} .
$$

So let us be given an $l \geq 1, f \in \mathscr{C}$, and $\varepsilon>0$. We know from [12, Theorem 4.2.4] that there is a $\delta$-fine triangulation $S_{1}, \ldots, S_{N}$ of $S$ where $\delta<1 / 3 l$ 
and $\left|f(x)-f\left(x^{\prime}\right)\right|<\varepsilon / 3$ if $\left|x-x^{\prime}\right| \leq \delta$. Consequently, $S_{j} \cap S_{j^{\prime}}=\varnothing$ whenever $x_{j} \in S_{j}, x_{j^{\prime}} \in S_{j^{\prime}}$, and $\left|x_{j}-x_{j^{\prime}}\right| \leq 1 / l$. Let $\mathscr{F}$ be the finite collection of all pairwise disjointed $\left\{S_{j_{1}}, \ldots, S_{j_{k}}\right\}$ and let $C$ be the set of all corners of the $S_{j}$ 's; i.e. $C$ is the $(0)$-skeleton of our triangulation. Given any $g: C \rightarrow \mathbb{R}^{m}$ we define its "affine" extension $\mathscr{E}(g): S \rightarrow \mathbb{R}^{m}$ by the simple request that $\mathscr{E}(g)=g$ on $C$ and that $\mathscr{E}(g)$ is affine on each of the simplexes $S_{j}, j \leq N$. Due to our choice of $\delta$, we have $\|f-\mathscr{E}(\tilde{f})\|_{\infty} \leq \varepsilon$ whenever $\left\|\left.f\right|_{C}-\tilde{f}\right\|_{\infty} \leq \varepsilon / 2$. Hence, our problem reduces to showing that for any $\left\{S_{j_{1}}, \ldots, S_{j_{k}}\right\}$ and typical $g: C \rightarrow \mathbb{R}^{m}$ the sets $M^{\prime}=\bigcap_{i=1}^{k} \mathscr{E}(g)\left(S_{j_{i}}\right)$ and $M_{i}^{\prime}=\mathscr{E}(g)^{-1}\left(M^{\prime}\right) \cap S_{j_{i}}$ are all $d_{k}$-dimensional polyhedra. For $i=1, \ldots, k$ let $\bar{x}_{0}^{i}, \ldots, \bar{x}_{n}^{i}$ be the corners of $S_{j_{i}}$; then the statement about $M^{\prime}$ follows immediately from Lemma 2. Since we also know (e.g. from the proof of that lemma) that typical $\left.\mathscr{E}(g)\right|_{S_{j_{i}}}$ is injective, we conclude that also each of the $M_{i}^{\prime}$ is a $d_{k}$-dimensional polyhedron.

After all this preparations it is now very easy to complete the

Proof of Theorem 1. First, note that

$$
\begin{aligned}
M_{k}(f)=\bigcup_{l=1}^{\infty}\left\{x^{1} ; \text { exists } x^{2}, \ldots, x^{k} \text { with } f\left(x^{1}\right)=\cdots=\right. & f\left(x^{k}\right), \\
& \left.\min _{i<i^{\prime}}\left|x^{i}-x^{i^{\prime}}\right| \geq \frac{1}{l}\right\} .
\end{aligned}
$$

Each of the sets on the right-hand side is obviously compact. Hence, $M_{k}(f)$ as well as $f\left(M_{k}(f)\right)$ are always of type $F_{\sigma}$. The upper estimate of the Hausdorff dimension of these set is only a weakening of the just proved Proposition 4. Finally, let $\left\{U\left(x^{l}, 2 r_{l}\right\}_{l=1}^{\infty}\right.$ be a base of topology in $B\left(0,1 ; \mathbb{R}^{n}\right)$. Then for any $l$ there is a diffeomorphism $\Phi$ of $B(0,1)$ onto itself with $\Phi\left(U\left(x^{l}, r_{l}\right)\right)=U(0,1 / 2)$ which induces the linear self-isometry $I_{\Phi}: f \rightarrow f \circ \Phi$ of $\mathscr{C}\left(B(0,1), \mathbb{R}^{m}\right)$. Since the class of sets of non- $\sigma$-finite $\mathscr{H}^{d_{k}}$-measure is invariant under $\Phi$, we obtain from Propositions 2 and 3 that for typical $f$ and all $l$ both $M_{k}(f) \cap U\left(x^{l}, r_{l}\right)$ and $f\left(M_{k}(f) \cap U\left(x^{l}, r_{l}\right)\right)$ are of non- $\sigma$-finite $\mathscr{H}^{d_{k}}$-measure (for $k=1$ only the second of these sets).

We have now finished the proof of the first theorem, so from now on there are no special assumptions about the number $k$ and also the projections $P, P^{\prime}, Q$, $Q^{\prime}$ become undefined.

We turn now to the second case, if the dimension of the target space does not exceed that of the source space. Here it seems to be slightly more convenient to study functions defined on the unit cube, but again we could take any bilipschitzly equivalent set.

Theorem 2. Let $n \geq m \geq 1$. Then for typical $f:[0,1]^{n} \rightarrow \mathbb{R}^{m}$ the following hold:

- $\operatorname{int}(\operatorname{im} f) \neq \varnothing, \partial(\operatorname{im} f)$ is of Hausdorff dimension $(m-1)$.

- For any $y \in \mathbb{R}^{m}$ the level set $f^{-1}(y)$ is of Hausdorff dimension at most $n-m$ and is of non- $\sigma$-finite $\mathscr{H}^{n-m}$-measure whenever $y \in \operatorname{int}(\operatorname{im} f)$.

Again, until the end of the proof of this theorem we will always assume $n \geq m \geq 1$. We split its contents into the two following propositions. 
Proposition 5. Let $\varphi$ be a Hausdorff function with $\lim _{r \searrow 0} \varphi(r) / r^{n-m}=0$. For typical continuous $f:[0,1]^{n} \rightarrow \mathbb{R}^{n}$ there exists a set $M \subset[0,1]^{n}$ such that $\mathscr{H}^{\varphi}(M)=0$ and $\operatorname{card}\left(f^{-1}(y) \backslash M\right) \leq m$ for each $y \in \mathbb{R}^{m}$.

Proof. For any $k \geq 1$ we introduce the open sets $\mathscr{G}_{k} \subset \mathscr{C}=\mathscr{C}\left([0,1]^{n}, \mathbb{R}^{m}\right)$ consisting of all functions $f$ for which there exist sets $M_{i}=M_{i}(f, k), i=$ $0, \ldots, m$, and an $\eta>0$ such that

$$
\begin{gathered}
\bigcup_{i=0}^{m} M_{i}=[0,1]^{n}, \quad \mathscr{H}_{\infty}^{\varphi}\left(M_{0}\right)<2^{-k}, \\
\left|f(x)-f\left(x^{\prime}\right)\right| \geq \eta \quad \text { if }\left|x-x^{\prime}\right|>\frac{1}{k} \text { and } x, x^{\prime} \in M_{i} \text { for some } i \geq 1 .
\end{gathered}
$$

Now, suppose that $f \in \bigcap_{k=1}^{\infty} \mathscr{G}_{k}$ and define $M=\lim \sup _{k \rightarrow \infty} M_{0}(f, k)$. Obviously, $\mathscr{H}_{\infty}^{\varphi}(M) \leq \lim _{k \rightarrow \infty} \sum_{j \geq k} \mathscr{H}_{\infty}^{\varphi}\left(M_{0}(f, j)\right)=0$; hence $\mathscr{H}^{\varphi}(M)=0$. Next, suppose that, for some $y \in \mathbb{R}^{m}, f^{-1}(y) \backslash M$ contains $m+1$ points $x_{1}, \ldots, x_{m+1}$ with $\min _{j<l}\left|x_{j}-x_{l}\right|>\delta>0$. We find a $k$ such that $x_{j} \notin \bigcup_{k^{\prime}>k} M_{0}\left(f, k^{\prime}\right)$ for each $j \leq m+1$. Then there is an $i \in\{1, \ldots, m\}$ and there are $j<l$ such that $x_{j}, x_{l} \in M_{i}(f, k)$. Therefore, $f \in \mathscr{G}_{k}$ implies $f\left(x_{j}\right) \neq f\left(x_{l}\right)$, contradiction. Consequently, the proposition is proved if we show that each $\mathscr{G}_{k}$ is dense in $\mathscr{C}$. So, let us be given any $f \in \mathscr{C}, k \geq 1$, and $\varepsilon>0$. We find an integer $N>\sqrt{n} k$ such that $\left|f(x)-f\left(x^{\prime}\right)\right|<\varepsilon / 2$ whenever $\left|x-x^{\prime}\right| \leq$ $\sqrt{n} / N$. Next, we choose an integer $p$ sufficiently large. Using the notation $S(N, t)=\{z \in[0,1] ;|N z-j| \leq t$ for some $j=0, \ldots, N\}$ and $\operatorname{GI}(x, t)=$ $\left\{j \in\{1, \ldots, n\} ; x_{j} \in S(N, t)\right\}$ for $t \in[0,1 / 2)$, we define the sets

$$
\begin{aligned}
M_{0} & =M_{0}(f, k)=\left\{x \in[0,1]^{n} ; \operatorname{card}\left(\mathrm{GI}\left(x, 2^{-p}\right)\right) \geq m\right\}, \\
M_{1} & =M_{1}(f, k)=\operatorname{cl}\left(\left\{x \in[0,1]^{n} ; \operatorname{card}\left(\mathrm{GI}\left(x, 2^{-p-1}\right)\right) \geq m-1\right\} \backslash M_{0}\right), \\
M_{2} & =M_{2}(f, k) \\
& =\operatorname{cl}\left(\left\{x \in[0,1]^{n} ; \operatorname{card}\left(\mathrm{GI}\left(x, 2^{-p-2}\right)\right) \geq m-2\right\} \backslash\left(M_{0} \cup M_{1}\right)\right), \\
& \vdots \\
M_{m-1} & =M_{m-1}(f, k) \\
& =\operatorname{cl}\left(\left\{x \in[0,1]^{n} ; \mathrm{GI}\left(x, 2^{-p-m+1}\right) \neq \varnothing\right\} \backslash\left(M_{0} \cup \cdots \cup M_{m-2}\right)\right), \\
M_{m} & =M_{m}(f, k)=\operatorname{cl}\left([0,1]^{n} \backslash\left(M_{0} \cup \cdots \cup M_{m-1}\right)\right) .
\end{aligned}
$$

Observe that for each $x \in M_{i}, i \geq 1, \mathrm{GI}(x, t)=\mathrm{GI}\left(x, t^{\prime}\right)$ and this set has precisely $i$ members whenever $t, t^{\prime} \in\left[2^{-p-i}, 2^{-p-i+1}\right)$. This implies that $\left|x-x^{\prime}\right| \geq 2^{-p-i} / N$ whenever $x, x^{\prime} \in M_{i}$ and $\mathrm{GI}\left(x, 2^{-p-i}\right) \neq \mathrm{GI}\left(x^{\prime}, 2^{-p-i}\right)$. Now suppose that $x, x^{\prime} \in M_{i}$ can be joined by a $\left(2^{-p-i} / N\right)$-chain; i.e. there is a sequence $\left\{x^{l}\right\}_{l=0}^{K} \subset M_{i}, x^{0}=x, x^{K}=x^{\prime}$, and $\left|x_{l}-x^{l+1}\right|<2^{-p-i} / N$ for all $l<K$. By the foregoing and induction $\operatorname{GI}\left(x, 2^{-p-1}\right)=\operatorname{GI}\left(x^{l}, 2^{-p-l}\right)$ for all $l$. Moreover, all $x_{j}^{l}$ belong to the same component of $S\left(N, 2^{p-i}\right)$ if $j \in \mathrm{GI}\left(x, 2^{-p-i}\right)$; otherwise all the $x_{j}^{l}$ are in the same component of $[0,1] \backslash S\left(N, 2^{-p-i}\right)$. In particular, each of the (finitely many) components of $M_{i}$ has diameter at most $\sqrt{n} / N$ (and the distance between two of them is at least $\left.2^{-p-i} / N\right)$. Hence, if we write $\mathscr{F}_{i}$ for the system of all components of $M_{i}, i \geq 1$, we can choose mutually different reals $a_{C}, C \in \mathscr{F}_{i}$, such that 
$\left|a_{C}-(f(z))_{i}\right|<\varepsilon / 4$ for some $z \in C$. Consequently, the choice of $N$ ensures the existence of a $g \in U(f, \varepsilon)$ fulfilling

$$
(g(x))_{i} \equiv a_{C} \text { for } x \in C \in \mathscr{F}_{i}, \quad i=1, \ldots, m .
$$

Obviously, we conclude that $g \in \mathscr{G}_{k}$ if we verify that $\mathscr{H}_{\infty}^{\varphi}\left(M_{0}\right)<2^{-k}$. But this follows from:

$$
\begin{aligned}
\mathscr{H}_{\infty}^{\varphi}\left(M_{0}\right) & \leq\left(\begin{array}{c}
n \\
m
\end{array}\right)(N+1)^{m} \mathscr{H}_{\infty}^{\varphi}\left(\left[-\frac{2^{-p}}{N}, \frac{2^{-p}}{N}\right] \times[0,1]^{n-m}\right) \\
& \leq\left(\begin{array}{c}
n \\
m
\end{array}\right)(N+1)^{m}\left(2^{p-1} N\right)^{n-m} \mathscr{H}_{\infty}^{\varphi}\left(\left[0,2^{1-p} / N\right]^{n}\right) \\
& \leq\left(\begin{array}{c}
n \\
m
\end{array}\right)(N+1)^{m} \frac{\varphi\left(\sqrt{n} 2^{1-p} / N\right)}{\left(\sqrt{n} 2^{1-p} / N\right)^{n-m}} \sqrt{n^{n-m}} \\
& <2^{-k} \text { for } p \text { large enough. }
\end{aligned}
$$

Proposition 6. Let an arbitrary set $M_{0} \subset \mathbb{R}^{m}$ be given. Then for typical continuous $f:[0,1]^{n} \rightarrow \mathbb{R}^{m}$ the following alternative is true: Either $M_{0} \backslash \operatorname{im} f \neq \varnothing$ or, for each $y \in M_{0}, f^{-1}(y)$ of non- $\sigma$-finite $\mathscr{H}^{n-m}$-measure.

Proof. Again (and for the last time) we play the Banach-Mazur game. We denote by $Q, Q^{\prime}$ the orthogonal projections of $\mathbb{R}^{n}$ onto $\mathbb{R}^{m}$ and onto its orthonormal complement $\mathbb{R}^{n-m}$, using the natural inclusion, so $Q+Q^{\prime}=\mathrm{Id}$. So suppose $U\left(f_{1}, \varepsilon_{1}\right)$ is the first "move" of A. If $M_{0} \backslash \operatorname{im} f_{1} \neq \varnothing$, then there is $y \in M_{0}$ with $\operatorname{dist}\left(y, \operatorname{im} f_{1}\right)>\delta>0$. Hence, $y \notin \operatorname{im} g$ for all $g \in U\left(f_{2}, \varepsilon_{2}\right)$ where $f_{2}=f_{1}$ and $\varepsilon_{2} \in\left(0, \min \left\{\varepsilon_{1}, \delta\right\}\right)$. So in this case, we win the game before it really starts. Therefore, we can assume $M_{0} \subset \operatorname{im} f_{1} ;$ in particular $M_{0}$ is bounded. First we choose $R_{1}^{\prime}$ such that $\left|f_{1}(x)-f_{1}\left(x^{\prime}\right)\right|+2 R_{1}^{\prime}<\varepsilon_{1} / 3$ whenever $\left|x-x^{\prime}\right| \leq 2 R_{1}^{\prime}$, and then choose a partition of $M_{0}$ into (nonvoid) pieces $M_{1}, \ldots, M_{n}$ all of diameter less than $R_{1}^{\prime}$. We also choose points $x^{i} \in f_{1}^{-1}\left(M_{i}\right)$ and.a positive $R_{1}<R_{1}^{\prime}$ such that the balls $B\left(x^{i}, 2 R_{i}\right)$ are all disjoint. Now we can find a function $f_{2} \in U\left(f_{1}, \varepsilon_{1}\right)$ such that $f_{2}(x)=f_{2}\left(x^{i}\right)+\left(2 R_{1}^{\prime} / R_{1}\right) Q\left(x-x^{i}\right)$ whenever $\left|Q\left(x^{i}-x\right)\right|,\left|Q^{\prime}\left(x-x^{i}\right)\right| \leq R_{1}$. To initialize our trees, we choose compact sets $K_{i} \subset B\left(Q^{\prime}\left(x^{i}\right), R_{1}\right)$ of positive $\mathscr{H}^{n-m}$-measure but projecting onto each of the coordinate axes into $\mathbb{R} \backslash \mathbb{Q}$. Then we put $\mathscr{K}_{0}^{i}=\left\{K_{i}\right\}, \mathscr{M}_{0}^{i}=\left\{M_{i}\right\}$, and $x\left[K_{i}, M_{i}, \varnothing\right]=x^{i}$. We return $U\left(f_{2}, \varepsilon_{2}\right)$ where $\varepsilon_{2}<R_{1} / 2$ is sufficiently small to make our answer admissible.

In Step II we obtain A's answer $U\left(f_{3}, \varepsilon_{3}\right)$; a standard degree argument similar to that used in the proof of Proposition 1 ensures that for any $i \leq N$, $K \in \mathscr{K}_{0}^{i}, M \in \mathscr{M}_{0}^{i}$, and an arbitrary $t \in K$ the inclusion

$$
\begin{gathered}
f_{3}\left(\left\{x ; Q^{\prime}(x)=t \text { and }|Q(x[K, M, \varnothing]-x)|<R_{1}\right\}\right) \\
\supset B\left(f_{2}(x[K, M, \varnothing]), R_{1}^{\prime}\right) \supset M
\end{gathered}
$$

holds. Again, we choose $R_{2}^{\prime}$ fulfilling $\left|f_{3}(x)-f_{3}\left(x^{\prime}\right)\right|+2 R_{2}^{\prime}<\varepsilon_{3} / 3$ provided $\left|x-x^{\prime}\right| \leq 2 R_{2}^{\prime}$. Next, for any $i \leq N$ we carry out the following construction: we choose partitions of $\mathscr{K}_{1}^{i}$ of $K_{i}$ and $\mathscr{M}_{1}^{i}$ of $M_{i}$, where $\mathscr{K}_{1}^{i}$ consists of closed sets and both partitions are $R_{2}^{\prime}$-fine, i.e. contain only members of diameter less than $R_{2}^{\prime}$. For each $(K, M) \in \mathscr{K}_{1}^{i} \times \mathscr{M}_{1}^{i}$ we choose $y[K, M] \in M$ and two different points $x[K, M, 0], x[K, M, 1] \in B\left(x\left[K_{i}, M_{i}, \varnothing\right], R_{1}\right)$ with

$$
Q^{\prime}(x[K, M, j])=Q^{\prime}\left(x\left[K_{i}, M_{i}, \varnothing\right]\right) \in K_{i}
$$


and

$$
\left|f_{3}(x[K, M, j])-y[K, M]\right|<\varepsilon_{3} / 4 \text { for } j=0,1 \text {. }
$$

We fix an $R_{2}<R_{2}^{\prime}, R_{1} / 4$ such that all the balls $B\left(x[K, M, j], 2 R_{2}\right)$ for $(K, M, j) \in \mathfrak{T}_{1}=\bigcup_{i=1}^{N} \mathscr{K}_{1}^{i} \times \mathscr{M}_{1}^{i} \times\{0,1\}^{1}$ become mutually disjoint. Similar to the first step, we get an $f_{4} \in U\left(f_{3}, 2 \varepsilon_{3} / 3\right)$ such that $f_{4}(x)=y[K, M]+$ $\left(2 R_{2}^{\prime} / R_{2}\right) Q(x-x[K, M, j])$ whenever $x \in C[K, M, j]=\left\{\bar{x} ; Q^{\prime}(\bar{x}) \in K\right.$ and $\left.|Q(\bar{x}-x[K, M, j])| \leq R_{2}\right\}$ and $(K, M, j) \in \mathfrak{T}_{1}$. Then we return $U\left(f_{4}, \varepsilon_{4}\right)$ with $\varepsilon_{4}=R_{2} / 2$. Since again $h\left\{x, Q^{\prime}(x)=t\right.$ and $\left.|Q(x-x[K, M, j])|<R_{2}\right\} \supset$ $B\left(y[K, M], R_{2}^{\prime}\right) \supset M$ for any $h \in U\left(f_{4}, R_{2} / 2\right), t \in K$, and $(K, M, j) \in \mathfrak{T}_{1}$, we can in Step III repeat the construction from Step II, but now on each of the "cylinders" $C[K, M, j]$.

In this way we obtain a declining sequence $R_{l+1}$ with $0<R_{l+2}<R_{l+1} / 4$; a nested sequence of open balls $U\left(f_{l+1}, \varepsilon_{l+1}\right) \in \mathscr{C}\left([0,1]^{n}, \mathbb{R}^{m}\right)$ with $\varepsilon_{2 l+2} \leq$ $R_{l+1}$; refining sequences $\mathscr{K}_{l}^{i}$ and $\mathscr{M}_{l}^{i}$ of $R_{l+1}$-fine partitions of the sets $K_{i}$ and $M_{i}$, resp., for $i \leq N$; and finally trees of points $x[K, M, \sigma] \in \mathbb{R}^{n}$, $(K, M, \sigma) \in \mathfrak{T}=\bigcup_{l=0}^{\infty} \mathfrak{T}_{l}, \mathfrak{T}_{l}=\bigcup_{i=1}^{N} \mathscr{K}_{l}^{i} \times \mathscr{M}_{l}^{i} \times\{0,1\}^{l}$ all of them for $l=0,1, \ldots$ and fulfilling:

$$
f_{2 l+3}\left(\left\{x ; Q^{\prime}(x)=t \text { and }|Q(x-x[K, M, \sigma])| \leq R_{l+1}\right\}\right) \supset M
$$

if $t \in K$ and $(K, M, \sigma) \in \mathfrak{T}_{l}, l \geq 0$.

$$
B\left(x\left[K^{\prime}, M^{\prime}, \sigma j\right], 2 R_{l+2}\right) \subset B\left(x[K, M, \sigma], 2 R_{l+1}\right)
$$

if $\left(K^{\prime}, M^{\prime}, \sigma j\right) \in \mathfrak{T}_{l+1},(K, M, \sigma) \in \mathfrak{T}_{l}, j=0,1, K^{\prime} \subset K$, and $M^{\prime} \subset M$.

$$
B\left(x\left[K^{\prime}, M^{\prime}, \sigma^{\prime}\right], 2 R_{l+1}\right) \cap B\left(x\left[K^{\prime \prime}, M^{\prime \prime}, \sigma^{\prime \prime}\right], 2 R_{l+1}\right)=\varnothing
$$

if $\left(K^{\prime}, M^{\prime}, \sigma^{\prime}\right),\left(K^{\prime \prime}, M^{\prime \prime}, \sigma^{\prime \prime}\right) \in \mathfrak{T}_{l}$ but different.

Let $f$ be the uniform limit of the $f_{l}$ 's. We finish as in the proof of Proposition 2 concluding that for any $i \leq N, y \in M^{i}, t \in K^{i}$ the set $S=f^{-1}(y) \cap Q^{\prime-1}(t)$ contains a perfect set.

Now we are prepared to finish the

Proof of Theorem 2. The fact that typically $\operatorname{int}(\operatorname{im} f) \neq \varnothing$ is based on the degree argument used already in the proof of foregoing Proposition 6. Given $f \in \mathscr{C}$ and $\varepsilon>0$ we find $g \in U(f, \varepsilon), \delta>0, z \in \mathbb{R}^{n-m}$, and $y \in \mathbb{R}^{m}$ such that $h\left(Q^{\prime-1}(z)\right) \supset U(y, \delta)$ for all $h \in U(g, \delta) . \mathscr{H}^{m-1}(\partial(\operatorname{im} f))>0$ is now obvious, since it holds for any boundary of a nonvoid open bounded set. (A more precise consideration would show again that this set typically does not have $\sigma$-finite $\mathscr{H}^{n-m}$-measure. However, we are mainly interested in its smallness.) The upper dimension estimate is only slightly more delicate. Take any $d>m-1$. Using again triangulation as in the proof of Proposition 4, we see that the piecewise affine functions (now on the unit simplex) which are injective on each face of the $(m)$-skeleton of their underlying triangulation form a dense set. Given such a function $f$ and a $\delta>0$ such that $\left|f(x)-f\left(x^{\prime}\right)\right| \geq \delta\left|x-x^{\prime}\right|$ whenever these points are on some common $m$-face, we obtain from the already well-known degree arguments that

$$
h(U(x, r)) \supset U\left(h(x), \frac{r \delta}{2}\right) \quad \text { if } 0<r<\operatorname{dist}(x,(m-1) \text {-skeleton })
$$

$$
\text { and } h \in U\left(f, \frac{r \delta}{4}\right) \text {. }
$$


Consequently, $\partial(\operatorname{im} h) \subset U(f(U((m-1)$-skeleton, $r), r \delta / 4))$ for all $h \in$ $U(f, r \delta / 4)$ and choosing $r$ sufficiently small we get the $H_{\infty}^{d}$-measure of the right arbitrarily small. This shows that for all $\varepsilon>0$ the set of $f$ with $\mathscr{H}_{\infty}^{d}(\partial(\operatorname{im} f))<\varepsilon$ contains an open subset dense in $\mathscr{C}$.

Since always $f^{-1}(y) \subset M \cup\left(f^{-1}(y) \backslash M\right)$ with $M$ chosen according to Proposition 5 for $\varphi(x)=x^{n-m} /(-\log x)$, we have $\operatorname{dim}_{\mathscr{H}} f^{-1}(y) \leq \operatorname{dim}_{\mathscr{H}} M \leq n-m$. Finally, let $\left\{U_{l}\right\}_{l=1}^{\infty}$ be a base of topology in $\mathbb{R}^{m}$. Then Proposition 6 implies that for typical $f$ and any $l \geq 1$ the level set $f^{-1}(y)$ is of non- $\sigma$-finite $\mathscr{H}^{n-m}$. measure whenever $y \in U_{l} \subset \operatorname{im} f$. Since such an $l$ exists for any $y \in \operatorname{int}(\operatorname{im} f)$, we are done.

We did not give a lower estimate for the size of level sets $f^{-1}(y)$ if $y \in$ $\partial(\operatorname{im} f)$. Indeed, by [5], for typical $f$ there are always points $y$ such that $f^{-1}(y)$ is a singleton.

Our last example will show that in the typical case no completely direct generalization of the results from [8] is possible. Indeed, together with Proposition 5 , it shows that for a typical continuous vector field on the unit square there is no set of injectivity with a zero-dimensional complement (although all level sets are zero dimensional), but that the largest sets of injectivity have a complement of Hausdorff dimension one with non- $\sigma$-finite measure.

Lemma 3. Let $f:[0,1]^{2} \rightarrow \mathbb{R}^{2}$ be continuous, let $M \subset[0,1]^{2}$ be of onedimensional measure zero, $c \in \mathbb{R}^{2}$, and let $B\left(x^{1}, R\right), B\left(x^{2}, R\right)$ be two disjoint balls such that

- $\operatorname{card}\left(f^{-1}(y) \backslash M\right) \leq 2$ for all $y \in \mathbb{R}^{2}$,

- $\left|f\left(x^{j}\right)-c-z\right|<R / 8$ for $j=1,2$ and $|z| \leq R$.

Then the restriction of $f$ to a set $[0,1]^{2} \backslash S$ is noninjective whenever $S$ is of $\sigma$-finite $\mathscr{H}^{1}$-measure.

Proof. Take any such $S \subset[0,1]^{2}$. Using the fact that the map $x \rightarrow\left|x-x^{2}\right|$ is lipschitz and Lemma 1 , we find a set $C \subset[3 R / 8,5 R / 8]$ of positive measure such that for any $t \in C$ the following hold:

- $\partial B\left(x^{2}, t\right) \cap M=\varnothing$,

- $\partial B\left(x^{2}, t\right) \cap S$ is countable,

- $f\left(\partial B\left(x^{2}, t\right)\right) \subset U(c, 6 R / 8) \backslash B(c, 2 R / 8)$.

Next, observe that the sets $\left(B\left(x^{1}, R\right) \backslash M\right) \cap f^{-1}\left(f\left(\partial B\left(x^{2}, t\right)\right)\right)$ are disjoint for $t$ 's different from $C$. Indeed, otherwise we would find $x \in B\left(x^{1}, R\right) \backslash M$ and different $x^{\prime}, x^{\prime \prime} \in B\left(x^{2}, R\right) \backslash M$ such that $f(x)=f\left(x^{\prime}\right)=f\left(x^{\prime \prime}\right)$-a contradiction to the choice of $M$. Since $\mathscr{H}^{1}(M)=0$ and $S$ is of $\sigma$-finite measure, we infer that for all except countably many and, therefore, at least for some $T \in C$

$$
\mathscr{H}^{1}\left(B\left(x^{1}, R\right) \cap f^{-1}\left(f\left(\partial B\left(x^{2}, T\right)\right) \cap S\right)\right)=0 .
$$

Because the map $x \rightarrow(x /|x|)$ is locally lipschitz on $\mathbb{R}^{2} \backslash\{0\}$, we conclude that the set $D$ of all $\theta \in[0,2 \pi)$ for which $\lambda e^{i \theta}+x^{1} \notin M \cup\left(f^{-1}\left(f\left(\partial B\left(x^{2}, T\right)\right) \cap S\right)\right)$ whenever $\lambda \in(0, R]$ is a set of full measure in $[0,2 \pi)$.

Moreover, since for all $\theta \in[0,2 \pi)$ both $f\left(e^{i \theta} R / 8+x^{1}\right) \in B(c, 2 R / 8)$ and $f\left(e^{i \theta} R+x^{1}\right) \notin U(c, 6 R / 8)$ hold and because the paths

$$
\gamma_{1}: \theta \rightarrow f\left(e^{i \theta} T+x^{1}\right) \text { and } \gamma_{2}: \theta \rightarrow e^{i \theta} R / 2+c
$$


are homotopically equivalent inside $U(c, 6 R / 8) \backslash B(2 R / 8)$, we conclude that $\operatorname{ind}\left(\gamma_{1}, f\left(e^{i \theta} R / 8+x^{1}\right)\right)=1 \neq 0=\operatorname{ind}\left(\gamma_{1}, f\left(e^{i \theta} R+x^{1}\right)\right)$. Therefore, for any $\theta \in[0,2 \pi)$ there are $\lambda_{\theta} \in[R / 8, R]$ and an $x^{\theta} \in \partial B\left(x^{2}, T\right)$ satisfying

$$
f\left(x^{\theta}\right)=f\left(e^{i \theta} \lambda_{\theta}+x^{1}\right) .
$$

Since $\partial B\left(x^{2}, T\right) \cap M=\varnothing$, we have $x^{\theta}, e^{i \theta} \lambda_{t h}+x^{1} \notin M$ for all $\theta \in D$. This shows that, for two different $\theta, \tilde{\theta} \in D, x^{\theta} \neq x^{\tilde{\theta}}$ holds. Because $S \cap \partial B\left(x^{2}, T\right)$ is countable, we conclude that $x^{\theta_{0}} \notin S$ for some $\theta_{0} \in D$. Since $\varepsilon^{i \theta_{0}} \lambda_{\theta_{0}}+x^{1} \notin S$ for all $\theta \in D$, we see that $f \mid\left([0,1]^{2} \backslash S\right)$ is noninjective indeed.

\section{REFERENCES}

1. A. M. Bruckner, Differentiation of real functions, Lecture Notes in Math., vol. 659, Springer, 1978.

2. A. M. Bruckner and K. M. Garg, The level structure of a residual set of continuous functions, Trans. Amer. Math. Soc. 232 (1977), 307-321.

3. A. M. Bruckner and G. Petruska, Some typical results on bounded Baire 1 functions, Acta Math. Hungar. 43 (1984), 325-333.

4. Yu. Burago and V. Zalgaller, Geometric inequalities, Grundlehren Math. Wiss., vol. 285, Springer-Verlag, 1988.

5. M. Chlebik, On extrema of typical functions (to appear).

6. K. Deimling, Nonlinear functional analysis, Springer-Verlag, 1985.

7. H. Federer, Geometric measure theory, Grundlehren Math. Wiss., vol. 153, Springer-Verlag, 1969.

8. B. Kirchheim, Some further typical results on bounded Baire one functions, Acta Math. Hungar. 62 (1993), 119-129.

9. _ Typical approximately continuous functions are surprisingly thick, Real Analysis Exchange 18 (1992-93).

10. J. Oxtoby, The Banach-Mazur game and Banach's category theorem, Contributions to the Theory of Games (Dresher, Tucker, Wolfe, eds.), Ann. of Math. Stud., no. 39, Princeton Univ. Press, 1957, pp. 159-163.

11. C. A. Rogers, Hausdorff measures, Cambridge Univ. Press, 1970.

12. I. M. Singer and J. A. Thorpe, Lecture notes on elementary topology and geometry, Scott, Foresman and Company, 1967.

Institute of Applied Mathematics, Comenius University, Mlynská Dolina, 84215 Bratislava, Slovakia tria

Current address: Institut für Mathematik, Johannes-Kepler Universität, Linz-Auhof, 4040 Aus-

E-mail address: kirchhei@dcs.fmph.uniba.sk 